\title{
Occupational Noise, Smoking, and a High Body Mass Index are Risk Factors for Age-related Hearing Impairment and Moderate Alcohol Consumption is Protective: A European Population-based Multicenter Study
}

Erik Fransen $^{1}$, Vedat Topsakal ${ }^{2}$, Jan-JaAp Hendrickx ${ }^{1,2}{ }^{2}$, Lut Van Laer ${ }^{1}$, Jeroen R. Huyghe ${ }^{1}$, Els Van Eyken ${ }^{1}$, Nele Lemkens ${ }^{2}$, Samuli Hannula ${ }^{3}$, Elina Mäki-Torkko ${ }^{3}$, Mona Jensen ${ }^{5}$, Kelly Demeester ${ }^{2}$, Anke Tropitzsch ${ }^{6}$, Amanda Bonaconsa ${ }^{7}$, Manuela Mazzoli ${ }^{7}$, Angeles Espeso ${ }^{8}$, Katia Verbruggen ${ }^{9}$, Joke Huyghe ${ }^{9}$, Patrick L. M. Huygen ${ }^{4}$, Sylvila Kunst ${ }^{4}$, Minna Manninen ${ }^{10}$, Amalia Diaz-Lacava ${ }^{11}$, Michael Steffens ${ }^{11}$, Thomas F. Wienker ${ }^{11}$, Ilmari Pyykkö ${ }^{10}$, Cor W. R. J. Cremers ${ }^{4}$, Hannie Kremer ${ }^{4}$, Ingeborg Dhooge ${ }^{9}$, Dafydd Stephens ${ }^{8}$, Eva Orzan ${ }^{7}$, Markus Pfister ${ }^{6}$, Michael Bille ${ }^{5}$, Agnete Parving ${ }^{5}$, Martti Sorri ${ }^{3}$, Paul Van de Heyning ${ }^{2}$, and Guy Van Camp ${ }^{1}$

${ }^{1}$ Department of Medical Genetics, University of Antwerp, Universiteitsplein, 2610 Antwerp, Belgium

${ }^{2}$ Department of Otorhinolaryngology, University Hospital of Antwerp, 2650 Antwerp, Belgium

${ }^{3}$ Department of Otorhinolaryngology, University of Oulu, 90014 Oulu, Finland

${ }^{4}$ Department of Otorhinolaryngology, St. Radboud University Medical Center, 6500 HB Nijmegen, The Netherlands

${ }^{5}$ Department of Audiology, Bispebjerg Hospital, 2400 NV Copenhagen, Denmark

${ }^{6}$ Department of Otorhinolaryngology, University of Tübingen, 72074 Tübingen, Germany

${ }^{7}$ Department of Oto-Surgery, University Hospital Padova, 35128 Padova, Italy

${ }^{8}$ College of Medicine, Cardiff University, CF14 4XW Cardiff, UK

${ }^{9}$ Department of Otorhinolaryngology, University Hospital of Ghent, 9000 Ghent, Belgium

${ }^{10}$ Department of Otorhinolaryngology, University of Tampere, 33014 Tampere, Finland

${ }^{11}$ Institute of Medical Biometry, Informatics and Epidemiology, University of Bonn, 53105 Bonn, Germany

Received: 4 December 2007; Accepted: 21 April 2008; Online publication: 10 June 2008

\begin{abstract}
A multicenter study was set up to elucidate the environmental and medical risk factors contributing to age-related hearing impairment (ARHI). Nine subsamples, collected by nine audiological centers across Europe, added up to a total of 4,083 subjects between 53 and 67 years. Audiometric data (pure-tone average [PTA]) were collected and the participants filled out a questionnaire on environmental risk factors and
\end{abstract}

Electronic supplementary material The online version of this article (doi:10.1007/s10162-008-0123-1) contains supplementary material, which is available to authorized users.

Correspondence to: Guy Van Camp ·Department of Medical Genetics University of Antwerp - Universiteitsplein 1, B-2610 Antwerp, Belgium. email: guy.vancamp@ua.ac.be medical history. People with a history of disease that could affect hearing were excluded. PTAs were adjusted for age and sex and tested for association with exposure to risk factors. Noise exposure was associated with a significant loss of hearing at high sound frequencies $(>1 \mathrm{kHz})$. Smoking significantly increased high-frequency hearing loss, and the effect was dosedependent. The effect of smoking remained significant when accounting for cardiovascular disease events. Taller people had better hearing on average with a more pronounced effect at low sound frequencies $(<2 \mathrm{kHz})$. A high body mass index (BMI) correlated with hearing loss across the frequency range tested. Moderate alcohol consumption was inversely correlated with hearing loss. Significant associations were found in the high as well as in the low fre- 
quencies. The results suggest that a healthy lifestyle can protect against age-related hearing impairment.

Keywords: age-related hearing impairment, presbyacusis, occupational noise, smoking, risk factors

\section{INTRODUCTION}

Several factors contribute to the decline in hearing acuity with increasing age. Apart from biological degeneration because of aging in itself, age-related hearing impairment (ARHI) is influenced by genetic risk factors, exposure to noise and toxic substances, and the occurrence of certain diseases. The relative contributions of the different risk factors are difficult to estimate, and the interactions between them remain unclear.

Typically, ARHI is sensorineural, bilaterally symmetrical and more pronounced at high frequencies with males more severely affected than females. There is a large variation between individuals, which is larger in males than in females (Lee et al. 2005). Variability increases with age and is greater at the high frequencies.

ARHI starts slowly around the fifth decade and worsens gradually, becoming the most common sensory impairment in the elderly. Between the ages of 60 and 70 years, about one third of the population has an average hearing loss (HL) of $25 \mathrm{~dB}$ or more for pure tones at $0.5,1,2$, and $4 \mathrm{kHz}$. Between the age of 70 and 80 years, the proportion of individuals with a pure-tone average (PTA) showing over $25 \mathrm{~dB}$ HL increases to $50 \%$ (Davis 1994). Although this is considered mild hearing loss, it seriously affects an individual's ability to communicate in a noisy environment.

\section{$Z$ scores}

When comparing ARHI in men and women of different ages, one needs to correct for gender and age differences between the subjects, and this correction is different depending on the frequencies studied. The expected median hearing threshold as a function of age, sex, and frequency, plus the standard deviation around this median, is given by the ISO 7029 standard (International Organisation of Standardization 1984). In a previous paper, we developed a method to quantify how severely a person is affected by age-related hearing loss, given his/her age and sex (Fransen et al. 2004). In this method, a $Z$ score is defined as the standardized difference between an individual's observed hearing threshold at a given frequency and the age- and sex-specific median for that frequency. This allows the comparison of individuals of different age and sex. Ideally, in a randomly collected highly screened population, $Z$ scores should have a standard normal distribution with no differences between males and females and no relation to age. However, several studies indicate that typical unscreened populations have slightly worse hearing than predicted by the ISO 7029 standard, whereby the former seem to have an apparent excess aging by $10-15$ years compared to the population described by the ISO 7029 standard (Robinson 1988; Lutman and Davis 1994; Engdahl et al. 2005).

\section{Importance of genetics}

The relative importance of genes in ARHI is agedependent. A heritability estimate by Karlsson et al. (1997) indicated that in the age stratum 56-65, 58\% of the variance in hearing thresholds was attributable to the influence of genes, declining to $47 \%$ in the stratum over 65. In the Framingham cohort, Gates et al. (1999) found a clear familial aggregation of the hearing thresholds. The heritability also seems to be frequency-dependent with a higher heritability in the low frequencies.

The advent of high-throughput methods for genetic analysis provides tools for identifying genetic variants implicated in ARHI. A genome-wide linkage study in the Framingham cohort identified several loci with suggestive evidence for linkage (DeStefano et al. 2003). Garringer et al. (2006) reported suggestive linkage in the DFNA18 region in the general population. Polymorphisms in the $N$-acetyltransferase 2 (NAT2) and KCNQ4 genes were found to be associated with ARHI in two independent populations (Ünal et al. 2005; Van Eyken et al. 2006, 2007).

\section{Environmental risk factors}

Whereas research into genetic variants associated with ARHI is still in its infancy, there is substantial literature about environmental risk factors contributing to ARHI. The influence of occupational noise is well-documented. The permanent threshold shift caused by exposure to occupational noise of a given intensity over a given period of time is predicted by the ISO 1999 standard (International Organisation of Standardization 1984). This effect is most pronounced at 2, 3, 4, and $6 \mathrm{kHz}$ (Dobie 2005).

However, individual noise susceptibility shows great variability. This may be because of complex interactions with nonoccupational noise, other environmental factors, or genetic predisposition (Helzner et al. 2005). The most deleterious source of nonoccupational noise is gunfire noise (Lutman and Spencer 1990; Clark 1991). People exposed to both occupational noise and gunfire noise have poorer hearing in both ears compared to people exposed to occupational noise alone (Stewart et al. 2001). 
A more than additive effect between noise and organic solvent exposure was found in a study of plastics factory workers coexposed to noise and styrene (Sliwinska-Kowalska et al. 2003), whereas an additive effect was observed in dockyard laborers coexposed to noise and a mixture of solvents (Sliwinska-Kowalska et al. 2004). Fuente and McPherson (2007) observed significantly increased hearing loss in workers exposed to a mixture of organic solvents compared to unexposed controls. Their results suggested solvent-induced central auditory damage.

There is controversy on the effect of smoking. Rosenhall et al. (1993) found an association between hearing levels and smoking; Cruickshanks et al. (1998) and Uchida et al. (2005) found indications of a dosage effect. On the other hand, no association was found in the Framingham cohort (Gates et al. 1993). Recent studies suggest an interaction between smoking and occupational noise, whereby the deleterious effect of noise exposure is exacerbated by smoking (Ferrite and Santana 2005; Nomura et al. 2005; Wild et al. 2005).

Data on alcohol consumption are not very clear. An association with chronic alcohol abuse has been observed (Rosenhall et al. 1993), but with moderate alcohol intake, the results are less clear. Brant et al. (1996) found no significant effect on the thresholds at speech frequencies but high frequencies were not tested. In contrast, Helzner et al. (2005) reported a protective effect at high frequencies, which depended on race and gender.

\section{Medical risk factors}

In addition to some environmental factors, several medical risk factors are suspected to have an influence on hearing. Many studies have focused on cardiovascular diseases (CVD), as these are very prevalent in the elderly population. In the Framingham cohort, an association between cardiovascular events (stroke, coronary heart disease, or intermittent claudication) and low-frequency hearing loss was reported (Gates et al. 1993). They also reported an inverse relationship between high-density lipoprotein (HDL) levels and hearing thresholds, suggesting a protective effect of HDL on hearing thresholds. Torre et al. (2005) found a significant association between myocardial infarction and hearing loss in females, but not in males. Brant et al. (1996) reported an association between hearing threshold and systolic blood pressure. A relationship between high-frequency sensorineural hearing impairment and diabetes mellitus has been reported by several investigators (Kurien et al. 1989; Vaughan et al. 2006), but this association is highly controversial because of the heterogeneity in diabetic patients. In a large cohort of Swedish conscripts, followed-up from birth till conscription (age 17-24), short adult stature and body mass index (BMI; either very high or very low) were found to be risk factors. These effects were dependent on birth weight and length (Barrenas et al. 2005). In particular, overweight subjects who were born light and small for their gestational age had an increased risk for hearing loss at conscription. In an adult Danish cohort, very short subjects between 50 and 55 years of age had a higher prevalence of hearing loss compared to very tall subjects (Burr et al. 2005).

This study is part of a European multicenter study into the genetic and environmental causes of ARHI. In this paper, we present data from a total of 4,083 unrelated subjects, collected through nine audiological centers from seven European countries. Potential risk factors were assessed by questionnaire for association with ARHI as established by audiometric criteria.

\section{METHODS}

\section{Sample collection}

The collection of subjects was a collaborative effort of nine expert audiological centers from seven European countries: two from Belgium (Antwerp, Ghent), two from Finland (Tampere, Oulu), one from The Netherlands (Nijmegen), one from Germany (Tübingen), one from Denmark (Copenhagen), one from Italy (Padua), and one from the UK (Cardiff). To collect study subjects, the audiological centers used three different recruitment strategies: (1) A clinic-based sample, whereby subjects are collected through the regular influx of patients visiting an audiological or ENT clinic. As this strategy tends to recruit an excess of people with poor hearing, the spouses of the recruited subjects were asked to join the study. (2) A population-based sample, whereby subjects were collected via advertisements in local media or through local population registers and letters of invitation. (3) A mixed strategy, whereby part of the samples was population-based and the remaining part was clinic-based. The nine sample sets collected by the audiological centers are hereafter referred to as subsamples.

To make each subpopulation ethnically homogeneous, we requested that at least three out of the four grandparents originated from the same region as the study subject. An effort was made to collect an approximately equal number of males and females and to have a uniform age distribution. All responding subjects underwent clinical examination and otoscopy and completed a detailed questionnaire on medical history and exposure to environmental risk factors. The complete questionnaire is available upon request. A list of all questions and answers used in this paper is provided in Supplementary Table 4. Subjects with ear diseases, possible monogenic forms of hearing impairment, or other major pathologies with a possible 
influence on hearing were excluded. The main goal was to study hearing impairment in healthy subjects and, therefore, persons with multiple hospitalizations were excluded. The complete list of exclusion criteria was previously reported (Van Eyken et al. 2006). In subjects passing the medical exclusion criteria, audiometric thresholds were determined for air conduction $(0.25,0.5,1,2,3,4,6$, and $8 \mathrm{kHz})$ and bone conduction $(0.5,1,2$, and $4 \mathrm{kHz})$ according to current clinical standards (ISO 8253). We excluded subjects with asymmetrical hearing loss (between-ear difference in air conduction threshold larger than $20 \mathrm{~dB}$ for at least two frequencies out of $0.5,1$, and $2 \mathrm{kHz}$ ). In case only one of the ears showed conductive hearing loss (air-bone gap of $15 \mathrm{~dB}$ or more at 0.5 , 1 , and $2 \mathrm{kHz}$ ) and in the absence of other exclusion criteria, the other ear could be included.

Research was approved by the ethical committees of the institutions connected to each research center: University of Antwerp, University Hospital of Antwerp, University of Oulu, University Medical Center Nijmegen, Bispebjerg Hospital Copenhagen, University of Tübingen, University Hospital Padova, Cardiff University, University Hospital of Ghent, University of Tampere, and University of Bonn. All persons gave their informed consent before inclusion in this study.

\section{$Z$ scores}

$Z$ scores were calculated as described by Fransen et al. (2004). In brief, for each individual, we calculated the age- and sex-specific median threshold at each frequency, based upon the ISO 7029 standard. This value was subtracted from the observed hearing thresholds at each frequency. The difference, which may be negative (=better hearing than median) or positive (=worse hearing than median), was normalized by dividing by the age-, sex-, and frequency-specific standard deviation given by the ISO 7029 standard. These calculations produce frequency-specific $Z$ scores. In this study, we used summary values for the high and low frequencies, respectively: The highfrequency $Z$ score $\left(Z_{\text {high }}\right)$ is the average of the $Z$ scores at 2,4 , and $8 \mathrm{kHz}$, and the low-frequency $Z$ score ( $Z_{\text {low }}$ ) is the average of the $Z$ scores at $0.25,0.5$, and $1 \mathrm{kHz}$. In all analyses presented in this study, we used $Z_{\text {high }}$ and $Z_{\text {low }}$ of the better hearing ear (based upon the average PTA at $0.5,1$, and $2 \mathrm{kHz}$ ).

\section{Exposure to gunfire noise}

The subjects were asked how many rounds they had fired with either light or heavy weapons. Rifles or machine guns were counted as light weapons, whereas large infantry weapons and artillery were counted as heavy weapons. For light weapons, five levels of noise exposure were used: $0=$ no exposure (never fired), $1=$ less than 100 rounds, $2=100-1,000$ rounds, $3=1,000$ 10,000 rounds, and $4=$ more than 10,000 rounds. For the heavy weapons, the latter two levels were combined to obtain an ordinal variable with four exposure levels. The use of hearing protection was documented, which allowed us to separately count shots with protection and shots without protection. Hence, every subject had four summary values describing the exposure to gunfire noise: protected light, unprotected light, protected heavy, and unprotected heavy.

To combine the cumulative exposure from the light and heavy weapons, we summed the exposure levels from light and heavy weapons, adding one to the exposure level of heavy weapons with an upper limit of four. This corresponds to multiplying the number of rounds with heavy weapons by ten and adding them to the number of light rounds.

\section{Hypertension}

Blood pressure was measured once according to standard procedures. These measurements were recoded into a binary variable to test for association with ARHI. Subjects with either a systolic blood pressure above $140 \mathrm{~mm} \mathrm{Hg}$ or a diastolic blood pressure above $90 \mathrm{mmHg}$ (Chobanian et al. 2003) or who were taking antihypertensive drugs were considered hypertensive.

\section{Statistical analysis}

To enable parametric data analysis (ANOVA or regression), Box-Cox transformations of the $Z$ scores were carried out using the statistical package $\mathrm{R}$ (http:// www.r-project.org). In each of the nine subsamples, a separate transformation was carried out, and all further calculations for that given subsample were performed on the transformed outcome variable. For the joint analysis, a new Box-Cox transformation on the combined dataset was carried out.

Association between the $Z$ scores and a categorical risk factor was tested using ANOVA. Ordinal or numeric risk factors were tested via linear regression.

Theoretically, in a highly screened population, $Z$ scores should correct for gender effects. However, we noticed that, in several of the subsamples, there was a significant difference in $Z$ scores between males and females. Therefore, gender was entered as a covariate into the regression or ANOVA models. All models were built in a stepwise backward way. First, a full model was fitted including the risk factor of interest, along with gender and the interaction term between these. In such model, the interaction term tests whether the effect of the risk factor is significantly different between the two sexes. If the interaction term was not significant, it was omitted from the 
model and a new model with only the two main effects was fitted. In case the gender term was not significant in this simplified model, it was omitted, resulting in a model containing only the risk factor. Otherwise, in cases for which the gender term was significant, we kept it in the model, and the significance of the risk factor was tested accounting for the gender effect.

To check the appropriateness of the fitted model and to find outlying observations, residual plots (normality of the raw residuals, predicted value vs. raw residuals, studentized residuals vs. independent variable) were visually inspected.

In the joint analysis, all nine subsamples were combined and a categorical variable for subsample was added to the model. This adjusted the estimated effect size and significance of the risk factors for differences in mean $Z$ scores between populations to obtain a common effect size.

To deal with the multiple testing burden, a Bonferroni correction was applied to the results of the joint analyses. A total of 86 tests were performed (Supplementary Table 3), 12 of which were part of a multivariable analysis and not independent from the univariable tests. Therefore, the number of independent tests equals 74 , and the threshold for significance was adjusted to $0.05 / 74=0.00068$.

\section{Cluster analysis}

We used the $p$ values of 43 association tests (in $Z_{\text {high }}$ and $Z_{\text {low }}$ ) to check whether the results obtained in the subsamples with the same recruitment strategy were more similar to each other than to the subsamples collected using a different strategy. A list of the 43 tests, corresponding to the tests shown in Supplementary Table 3, is given in Supplementary Table 5.

For the cluster analysis, the $p$ values of the 43 tests in the nine subsamples were arranged in a $9 \times 43$ matrix. Hence, each subsample was considered to be one 43variate observation. Hierarchical clustering (complete linkage) was performed using the cluster package in R. The image plots were generated using the fields package in $\mathrm{R}$.

\section{RESULTS}

\section{Collection of subjects}

Through nine clinical (ENT/audiological medicine) groups, unrelated Caucasian subjects were collected in seven different European countries. Table 1 lists the collecting groups, the sizes of the subsamples, and the recruitment strategy.

A total of 4,083 subjects, including 1,967 males and 2,116 females, passed our inclusion criteria. The age range was set at 53 to 67 years. The mean overall age was 60.4 for females $(\mathrm{SD}=3.2)$ and 60.9 for males $(\mathrm{SD}=$ 3.2). The mean ages for males and females for each country and center are given in Supplementary Table 1. Mean audiograms, showing the mean thresholds and standard deviation at each frequency, are shown in Supplementary Fig. 1. The mean $Z$ scores for each subsample are shown in Supplementary Table 2.

\section{Association testing}

Most questions from the questionnaire refer to previously reported environmental and medical risk factors. We have tested these risk factors for association with hearing loss in the high and low frequencies. To compare the hearing acuity in subjects with a different age and gender, audiometric measurements were converted to $Z$ scores.

For each of the risk factors, we first analyzed all nine subsamples separately. We have looked for significant associations that replicated across multiple subsamples with all associations having the same direction. Afterwards, a joint analysis was performed on all nine subsamples combined. The Bonferroni-adjusted significance threshold for the joint analysis was 0.00068 .

\section{TABLE 1}

\begin{tabular}{llcccc}
\hline \multicolumn{5}{c}{ TABLE 1 } \\
\hline City & \multicolumn{5}{c}{} \\
\hline Antwerp & Country & Males & Females & Total & Recruitment \\
Copenhagen & Belgium & 556 & 606 & 1,162 & Population registries \\
Cardiff & Denmark & 212 & 208 & 420 & Clinic \\
Gent & United Kingdom & 197 & 159 & 356 & Clinic \\
Nijmegen & Belgium & 159 & 179 & 338 & Clinic+advertisement \\
Oulu & Netherlands & 146 & 138 & 284 & Clinic \\
Padua & Finland & 218 & 282 & 500 & Population registries \\
Tampere & Italy & 173 & 189 & 362 & Clinic+advertisement \\
Tübingen & Finland & 100 & 155 & 255 & Clinic \\
Total & Germany & 206 & 200 & 406 & Clinic+population registries \\
& & 1,967 & 2,116 & 4,083 &
\end{tabular}


TABLE 2

Association test between $Z$ score and ARHI risk factors in subsamples and joint analysis

\begin{tabular}{|c|c|c|c|c|c|c|c|c|c|c|c|}
\hline Risk factor ${ }^{a}$ & Answer & Antwerp & Cardiff & Copenhagen & Ghent & Nijmegen & Oulu & Padua & Tampere & Tübingen & Joint analysis ${ }^{b}$ \\
\hline \multicolumn{12}{|l|}{ Morphometry } \\
\hline Height & Number & & & & & & & & & & \\
\hline$Z_{\text {high }}$ & & & & & & 0.04 & & & & 0.009 & 0.05 \\
\hline$Z_{\text {low }}$ & & $5.4 \mathrm{E}-5$ & & 0.05 & & 0.02 & 0.03 & 0.007 & 0.02 & & $3.5 E-7$ \\
\hline BMI & Number & & & & & & & & & & \\
\hline$Z_{\text {high }}$ & & 0.03 & & & & & 0.08 & & 0.08 & $7.7 \mathrm{E}-7$ & 0.0004 \\
\hline$Z_{\text {low }}$ & & $6.3 \mathrm{E}-8$ & & 0.06 & & & & & 0.07 & $4.0 \mathrm{E}-5$ & $2.7 E-7$ \\
\hline \multicolumn{12}{|l|}{ Medical risk factors } \\
\hline Heart attack & Yes/no & & & & & & & & & & \\
\hline$Z_{\text {high }}$ & & & & & - & - & & - & - & - & 0.05 \\
\hline$Z_{\text {low }}$ & & & & 0.02 & - & - & 0.03 & - & - & - & 0.002 \\
\hline CVD event & Yes/no & & & & & & & & & & \\
\hline$Z_{\text {high }}$ & & & & & - & 0.09 & & - & - & - & \\
\hline$Z_{\text {low }}$ & & & & 0.02 & - & 0.01 & 0.048 & - & - & - & 0.0003 \\
\hline \multicolumn{12}{|l|}{ Noise and solvent exposure } \\
\hline Gunfire noise & Number & & & & & & & & & & \\
\hline$Z_{\text {high_prot }}$ & & 0.01 & & & & & & & & & \\
\hline$Z_{\text {high_unpr }}$ & & & & & 0.003 & & & & & & 0.009 \\
\hline Occupational noise exposure & Yes/no & & & & & & & & & & \\
\hline$Z_{\text {high }}$ & & & 0.01 & 0.0005 & 0.01 & $3.0 \mathrm{E}-5$ & 0.0002 & 0.01 & 0.002 & 0.001 & $1.0 E-17$ \\
\hline$Z_{\text {low }}$ & & & 0.007 & 0.01 & & 0.01 & & & 0.02 & 0.04 & $1.0 E-9$ \\
\hline Years noise exposure & Number & & & & & & & & & & \\
\hline$Z_{\text {high }}$ & & 0.09 & 0.02 & 0.007 & & 0.0003 & 0.0005 & 0.002 & 0.002 & 0.005 & $1.0 E-17$ \\
\hline$Z_{\text {low }}$ & & & 0.07 & & & 0.06 & & & 0.02 & 0.06 & $1.1 E-7$ \\
\hline Solvent exposure & Yes/no & & & & & & & & & & \\
\hline$Z_{\text {high }}$ & & & & & & & 0.07 & & & 0.07 & 0.001 \\
\hline$Z_{\text {low }}$ & & 0.07 & & & & & & & & & \\
\hline \multicolumn{12}{|l|}{ Smoking } \\
\hline Current or former smoking & Yes/no & & & & & & & & & & \\
\hline$Z_{\text {high }}$ & & & 0.01 & & 0.01 & & 0.03 & & & & 0.0009 \\
\hline$Z_{\text {low }}$ & & 0.02 & 0.06 & & & & & & & & 0.08 \\
\hline $\begin{array}{l}\text { Smoking history } \\
\text { (packyears) }\end{array}$ & Number & & & & & & & & & & \\
\hline$Z_{\text {high }}$ & & 0.053 & 0.001 & 0.02 & 0.006 & 0.0200 & 0.0002 & & & & $1.0 E-9$ \\
\hline$Z_{\text {low }}$ & & & 0.03 & & & & & & & & 0.0004 \\
\hline $\begin{array}{l}\text { Packyears by sex } \\
\text { (high frequency) }\end{array}$ & Number & & & & & & & & & & \\
\hline Male & & & 0.02 & 0.01 & 0.08 & 0.03 & 0.0004 & & & & $1.9 E-7$ \\
\hline Fem & & & 0.02 & & 0.02 & & & & & & 0.0006 \\
\hline $\begin{array}{l}\text { Packyears by sex } \\
\text { (low frequency) }\end{array}$ & Number & & & & & & & & & & \\
\hline Male & & & 0.07 & & & 0.03 & & & & & 0.001 \\
\hline Female & & 0.04 & & & & & & & & & 0.07 \\
\hline $\begin{array}{l}\text { Smoking adjusted for CVD } \\
\text { and BMI }\end{array}$ & Number & & & & & & & & & & \\
\hline$Z_{\text {high }}$ & & 0.03 & 0.003 & 0.01 & 0.01 & 0.06 & 0.0006 & & & & $2.4 E-8$ \\
\hline$Z_{\text {low }}$ & & & 0.07 & & & & & & & & 0.008 \\
\hline $\begin{array}{l}\text { Smoking dosage effect } \\
\text { in smokers }\end{array}$ & Number & & & & & & & & & & \\
\hline$Z_{\text {high }}$ & & 0.09 & 0.04 & 0.001 & & & 0.006 & & & & $3.0 E-7$ \\
\hline$Z_{\text {low }}$ & & & & 0.08 & 0.08 & & & & & & 0.005 \\
\hline \multicolumn{12}{|l|}{ Alcohol consumption } \\
\hline Alcohol consumption & Yes/no & & & & & & & & & & \\
\hline$Z_{\text {high }}$ & & 0.06 & & 0.01 & & 0.002 & 0.050 & & & 0.09 & $8.4 E-6$ \\
\hline$Z_{\text {low }}$ & & & & & 0.01 & 0.002 & & & & 0.006 & $5.2 E-6$ \\
\hline
\end{tabular}

Only significant $(p<0.05)$ associations or trends toward significance $(p<0.1)$ are shown, other cells are empty. " - " means there were not enough exposed subjects to test the risk factor.

${ }^{\mathrm{a}}$ An extended table with all associations tested is provided as Supplementary Table 3. The exact questions and answers as they appeared in the questionnaire are provided as Supplementary Table 4.

${ }^{b}$ Values in italics remain significant after Bonferroni correction for 74 tests $(p<6.8 \mathrm{E}-4)$. 
Table 2 lists the associations that were significant $(p<$ $0.05)$ or showed a trend towards significance $(p<0.1)$ and also showed a significant association in the joint analysis. A complete overview of all associations tested is provided in Supplementary Table 3.

\section{Morphometry}

Besides environmental and medical risk factors, some studies have reported associations with morphometric characteristics. In our nine subsamples, we found several associations between $Z$ scores and body weight and the BMI. The associations were most pronounced for BMI. In all subsamples with a significant association (2/9 for $Z_{\text {high }}$ and $Z_{\text {low }}$ ), as well as in the two further subsamples with a trend toward association $(p<0.1)$, a higher body weight or BMI leads to a significant increase in the mean $Z$ score. Because a higher $Z$ score reflects worse hearing, this means that people with a higher BMI on average have more hearing loss. Upon joint analysis, BMI was highly significantly associated with both $Z_{\text {high }}$ and $Z_{\text {ow, }}$ whereas body weight was only associated with $Z_{\text {low }}$. As the effect of BMI is possibly because of confounding with CVD and smoking, we performed a multivariable analysis that included all three risk factors (Supplementary Table 3). The significance of the association between $Z$ scores and BMI slightly diminished when controlling for the two other risk factors, but the joint analysis was still highly significant.

No associations were found between $Z$ scores and eye color, and there was only weak evidence for an association with sunburn susceptibility and left/righthandedness. Body length was associated with $Z_{\text {low }}$ in 5/9 subsamples and with $Z_{\text {high }}$ in 2/9 subsamples. Across all significant associations, we observed that a higher body length was negatively associated with the $Z$ score. This implies that taller people on average have better hearing.

\section{Medical risk factors}

Compared to the Framingham study (Gates et al. 1993), the ARHI study showed a very low prevalence of common diseases (Table 3). This is probably attributable to the lower age range of the ARHI study compared to the mean age of the Framingham subjects (age 73) in addition to the exclusion of subjects with multiple hospitalizations in the ARHI study.

In this study, we tested whether a number of common diseases lead to increased $Z$ scores. We only tested for association in a subsample if the number of patients with the disease was sufficiently large (at least 15). The data on medical history were solely based on the questionnaire and no validation has been performed. Therefore, the association results on medical risk factors have to be regarded with caution.
TABLE 3

Prevalence of common diseases and smoking in ARHI and Framingham study

\begin{tabular}{lcc}
\hline Risk factor & ARHI study & Framingham study \\
\hline Smoking & & \\
Total number of subjects & 4,102 & 1,665 \\
Percentage exposed & 47.3 & 13.5 \\
Heart attack & & \\
Total number of subjects & 4,078 & 1,662 \\
Percentage affected & 3 & 10.4 \\
Intermittent claudication & & \\
Total number of subjects & 4,074 & 1,662 \\
Percentage affected & 1 & 7.2 \\
Stroke & & \\
Total number of subjects & 4,075 & 1,664 \\
Percentage affected & 0.1 & 7 \\
Total CVD & & 1,664 \\
Total number of subjects & 4,083 & 27.8 \\
Percentage affected & 2.0 & 1,664 \\
Coronary heart disease & & 19.4 \\
Total number of subjects & 4,083 & \\
Percentage affected & 1.1 & \\
\hline
\end{tabular}

Either no or only very weak evidence for association was found for hypertension, whiplash injuries, osteoporosis, osteoarthritis, allergy, or diabetes (Supplementary Table 3). CVD has often been reported as a risk factor for hearing loss, but in most of our subsamples, the number of affected individuals was too small to test for association with the $Z$ scores. We could test four subsamples for an association between hearing loss and heart attack. In two of them, the association with $Z_{\text {low }}$ was significant in a way that people having had a heart attack had on average an increased $Z_{\text {low }}$ compared to the rest of the subjects. The joint analysis where a larger number of subjects were available showed a nominally significant association with lowfrequency hearing loss. To test whether the occurrence of any CVD event is a risk factor for hearing loss, we created an indicator variable denoting the occurrence of either coronary heart disease, heart attack, stroke, or intermittent claudication. Subjects having taken medicine for any of these disorders were also considered affected. In three subsamples (of the five we could analyze), we found an association between this indicator variable and $Z_{\text {low. }}$. This significant association with $Z_{\text {low }}$ was also observed upon joint analysis (Table 2), reaching the Bonferroni-corrected significance threshold.

\section{Influence of drugs}

The use of some drugs may have an influence on hearing ability. Only in the Tampere subsample, aspirin use was associated with increased $Z_{\text {high }}$, but the 
association was not very strong $(p=0.03$, Supplementary Table 3). The use of painkillers, on the other hand, was associated with increased hearing loss in the low frequencies in the Antwerp and Tübingen subsamples, but the joint analysis did not reach significance $(p=0.06$, Supplementary Table 3$)$. Only in the Cardiff subsample were we able to test the influence of atorvastatin, but no significant effect was found (Supplementary Table 3).

\section{Gunfire noise}

To score the exposure to gunfire noise, we asked the subjects how many rounds of ammunition they had fired with either light or heavy weapons with and without hearing protection. A combined exposure level for light and heavy weapons was calculated, separately counting the rounds fired with and without protection, so that each individual had an unprotected and a protected exposure level.

To test the influence of gunfire exposure on hearing, we regressed the $Z$ scores from the best ear on the protected and unprotected gunfire exposure levels. Only two significant associations were found for $Z_{\text {high }}$ in two separate subsamples: in the Ghent subsample, there was a significant association with the unprotected exposure level $(p=0.003)$; the other association was with the protected exposure level in the Antwerp subsample $(p=0.01)$. Upon joint analysis, we found an association only between $Z_{\text {high }}$ and the unprotected exposure level (Table 2). In a multivariable analysis that also included exposure to occupational noise, the effect of gunfire noise showed no consistent effect across the subsamples, and the overall effect was only marginally significant in the high frequencies.

\section{Leisure noise}

Very low numbers of subjects reported repeated exposure to noise during their leisure time, and the time they had been exposed varied considerably. Therefore, the effect of leisure noise was not analyzed further.

\section{Occupational noise}

Work histories of the subjects were collected. As the subpopulations were collected without any selection regarding occupational noise, there was a large variation in the number of jobs with noise exposure, the total number of years worked in noise, and the age of the individuals at the time of exposure. To classify the subjects according to occupational noise exposure, we asked every subject whether (s) he had ever worked for more than a year in a noisy environment and whether a raised voice was necessary to communicate to a colleague at $1 \mathrm{~m}$ distance. In addition, we asked for the duration of the exposure and whether ear protection was used.

In all subsamples except the one from Antwerp, we found a significant association between occupational noise exposure and high-frequency hearing loss. Regardless of the duration of the exposure and of the use of protection, we consistently observed that people having worked for more than 1 year in a noisy place had higher $Z$ scores in the high frequencies compared to unexposed people. The same trend, but smaller, was observed in the lower frequencies. Significance was reached in 5/9 subsamples (Table 2).

Quantifying the noise exposure by calculating the number of years exposed and the daily exposure time also showed significant and consistent associations for hearing loss in both high and low frequencies, but the significance level was slightly decreased compared to the previous analysis. Accounting for the exposure to gunfire noise did not have much influence on the significance of the association between $Z_{\text {high }}$ and occupational noise. In the low frequencies, a slight increase in significance was observed.

Only $16 \%$ of exposed subjects reported using hearing protection 'always' or 'most of the time'. Restricting our analysis to exposed males, only four of the subsamples offered sufficient numbers to test for an effect of protection on hearing impairment. Only the Oulu subsample showed a significant protective effect in the high-frequency range $(p=0.02)$. Upon joint analysis, we also saw a significant effect in high frequencies ( $p=0.04$, Supplementary Table 3). Although the significance is marginal, this $p$ value was reached with very low numbers: of all noiseexposed males only $110(12 \%)$ wore protection most of the time or always.

\section{Solvents and toxic chemicals}

Subjects were asked for occupational exposure to organic solvents and other toxic substances. We had enough exposed individuals for statistical testing only for organic solvents, including aromatic carbohydrates (toluene, xylene, and styrene), trichloroethylene, and hexane. The number of people exposed to other substances was too small to test. As only 13 females were exposed to solvents, we restricted this analysis to males.

When organic solvent exposure was scored as a binary trait, none of the subsamples showed a significant association with hearing levels, although two subsamples showed a trend in the high frequencies. The joint analysis gave a nominally significant association (not reaching the Bonferroni-corrected significance level) in the high frequencies, which may imply that the negative result in the subsamples is merely 
because of the small number of exposed subjects and that there indeed may be a small effect that only becomes significant if the numbers are high enough (Table 2).

\section{Noise-solvent interaction}

We tested for nonadditive effects of noise and solvent exposure because synergistic effects between these two risk factors have been reported. In none of the subsamples did we find a significant interaction between noise and solvents. The group of people exposed to both occupational noise and solvents did not show significantly increased hearing loss compared with unexposed subjects or subjects exposed to only one of the two risk factors alone (Supplementary Table 3). The numbers are quite small, however, and the lack of significance may merely reflect the lack of power of this study to detect small interaction effects.

\section{Smoking}

Subjects were asked about smoking habits by first inquiring as to whether they had ever smoked regularly. In addition, we asked for the number of years they had been smoking and the number of cigarettes per day.

Dichotomizing the population into ever-smokers and never-smokers showed a significant association with $Z_{\text {high }}$ in 3/9 subsamples and with $Z_{\text {low }}$ in $1 / 9$ subsamples (Table 2). To investigate dosage effects, we estimated the number of packyears by multiplying the time (in years) an individual had been smoking by a weighting factor for daily consumption of tobacco $(<10$ cigarettes per day $=0.5 ; 10-20$ cigarettes per day $=1 ;>20$ cigarettes per day $=1.5)$. For nonsmokers, the number of packyears was set to zero. Linear regression revealed significant associations between packyears and $Z_{\text {high }}$ in 5/9 subgroups. The effect was less pronounced at the low frequencies with only one significant association (in the Cardiff subsample), but the joint analysis was highly significant for both the high and the low frequencies.

In none of the subsamples did we find a significant interaction between smoking and gender. Previous studies had tested for association in males and females separately. With this latter association test, the most significant associations were found in males in the high frequencies (4/9 subsamples significant; Table 2, packyears by sex). Among the five subsamples that initially showed a significant main effect of packyears, three subsamples no longer showed significant values in women, whereas the males remained significant. The opposite was observed in one subsample (Ghent), whereas in the fifth subsample, both sexes remained significant.

When restricting the analysis to smokers, we saw a significant dose effect in 3/9 subsamples at the high frequencies (Table 2, smoking dosage effect). Among smokers, there was a significant association between the number of packyears and high-frequency hearing loss. These analyses were highly significant on joint analysis. The higher significance when testing the association with packyears gives support to the hypothesis that the effect of smoking on hearing loss is dose-related.

The association between smoking and hearing loss remained highly significant when accounting for CVD events and BMI. There were still 5/9 subsamples having a significant effect of smoking on $Z_{\text {high }}$ with only minor changes in significance level. This multivariable analysis shows that the association between smoking and $Z$ scores cannot be attributed to confounding with CVD events.

\section{Noise-smoking interaction}

In the male subjects, we tested for nonadditive effects of occupational noise and smoking on hearing. $Z_{\text {high }}$ and $Z_{\text {low }}$ were regressed on the binary variables for smoking and occupational noise and the interaction between them. In one of the subsamples (results not shown), a weakly significant interaction was found ( $p=$ $0.04)$, but this effect was not found upon joint analysis $(p>0.05)$. Therefore, we did not find strong evidence that the effect of noise on hearing may be different between smokers and nonsmokers. Subdividing the population into four groups (noise exposure, smoking, both noise and smoking, or none) did not indicate a more-than-additive effect of noise and smoking (Supplementary Table 3). Just as in the case of the noisesolvent interaction, this study does not have high power to detect interactions. It should be noted that the lack of significance in the interaction tests may merely reflect a lack of power.

\section{Alcohol consumption}

Subjects were asked if they regularly (at least once a week) consumed alcohol. One glass of wine, spirit, or beer counted as one unit of consumption. Analyzing alcohol consumption as a binary variable showed significant association in $3 / 9$ subsamples in the high as well as in the low frequencies (Table 2). In addition, two further subsamples (Antwerp and Tübingen) showed a trend in the high frequencies $(p<0.1)$. In all these analyses, alcohol consumption consistently leads to a decrease in $Z$ scores and, thus, to improved hearing. Contrary to previous reports, we found no 
evidence for the effect being modified by gender (not shown).

\section{DISCUSSION}

The study presented in this paper is part of a study into the genetic, environmental, and medical causes of ARHI across seven European countries. In this study, we have used the results of a questionnaire to study the association between hearing levels and several putative environmental and medical risk factors. The use of questionnaire data implies the study is retrospective. No further validation of any questionnaire result has been performed.

The subjects in our study are between 53 and 67 years of age, which is relatively young to study this pathology. There are several reasons for choosing this age range.

First, this study is not only focused on the hearing loss because of aging alone (occurring in the absence of risk factors), but also on the influence of risk factors at earlier (preretirement) age. Although we had the long list of medical and otological exclusion criteria, people with noise or solvent exposure were not excluded. Hence, the hearing loss in our study population is because of a combination of exposures and aging. Second, we are using this sample set to find genes involved in ARHI, and the relative importance of genes is higher in younger age groups. Results of the genetic studies are published elsewhere (Van Laer et al. 2008). Third, collecting relatively young persons offers the potential for follow-up and longitudinal data analysis. This is very relevant in view of the ongoing debate about the influence of early noise exposure on hearing loss later in life. Studies in mice indicate that early noise damage can render the cochlea more vulnerable for hearing loss at later ages (Kujawa and Liberman 2006), whereas studies in human show that a cochlea that was previously damaged by noise (i.e., showing an audiometric notch) has a different pattern of age-related hearing loss later in life compared to an undamaged cochlea (Gates et al. 2000; Lee et al. 2005). A cross-sectional study like the one presented in this paper has weak power to disentangle the effects of early exposures and aging, but the dataset offers the potential to study this in the future.

For every risk factor, we first tested association in nine subsamples separately, followed by a joint analysis on the combined sample. Not all association tests showing a $p$ value below 0.05 represent a genuine effect. We have performed a large number of independent tests (a total of 566 univariable tests in the subsamples and 74 joint analysis tests, Supplementary Table 2). Even if no effect is present, many nominally significant associations will be observed just by chance. A classic way to overcome this multiple testing problem is to divide the threshold for statistical significance by the number of independent tests. This approach (the Bonferroni method) fixes the familywise error rate to $5 \%$, but is known to be conservative. In fact, when we apply this method to the tests in the subsamples, only a few associations (with a $p$ value below $0.05 / 566=8.8 \mathrm{E}-5$ ) would remain significant. The joint analyses reach higher significance levels because of the larger sample size. As only 74 independent joint tests were performed, the Bonferronicorrected significance threshold is 0.00068 , and 21 of the association tests reached significance. The joint association tests reaching Bonferroni-corrected significance are marked in italics in Table 2 and marked in bold in Supplementary Table 3.

The joint analysis is very powerful to detect small effects that cannot be picked up in the separate subsamples. In a heterogeneous sample like the ARHI sample, it is possible that the results of the subsamples differ because of their different recruitment strategies. Therefore, we have included a covariate for origin in the joint analysis to avoid a spurious association because of confounding factors that differ between subsamples. Still, it is useful to consider the results of the separate subsamples. A consistent effect in many of the subsamples, in addition to an overall effect, is a strong indication that the effect is genuine, even if only borderline significance levels are observed in the subsamples.

The nine subsamples were collected using three different recruitment strategies (Table 1), which may bias the sample. We have tested whether the recruitment strategy has an influence on the results of the analysis, but no indications of this were found. First, cluster analysis on the $p$ values did not indicate that the results obtained in the subsamples with the same recruitment strategy were more similar to each other than to subsamples collected using a different strategy (Supplementary Fig. 2). Second, visual inspection of the significance levels of the different association tests using an image plot (Supplementary Fig. 3) did not suggest any relation between recruitment strategy and significance level.

Exposure to occupational noise was by far the most significant and consistently replicated risk factor. Although the subjects in our sample sets were not selectively sampled for this type of analysis, and exposure levels, the duration, and the type of noise (impulse noise or steady noise) were highly heterogeneous, the association with high-frequency hearing loss was significant in all but one subsample. The effect at low frequencies was also consistent, although less pronounced. It is interesting to note that the effect on the low frequencies becomes more significant when gunfire noise is taken into account. 
Noise exposure has been reported to act synergistically with exposure to organic solvents. We found no evidence for noise-solvent interactions, but this is most likely because of the small number of people in the doubly exposed group, which allows the detection of only major nonlinear effects. The interaction tests in our study lacks power to detect small interaction effects, and not finding a significant interaction does not mean it does not exist. For solvent exposure alone, on the other hand, the joint analysis showed a significant effect in the high frequencies, despite the relatively low number of exposed subjects and the heterogeneity in exposure time and constitution of the solvent.

Our study conflicts with a previous report on the effect of smoking on hearing loss (Gates et al. 1993). In this study, we observed a highly significant association in more than half of our subsamples, consistently indicating a deleterious effect on high-frequency hearing. It is interesting to note that the effect was most pronounced in a quantitative analysis taking into account the number of packyears, rather than dichotomizing the population into people who ever smoked and people who never did. This is in line with previous reports of a dosage effect (Gates et al. 1993). A more specific analysis of this dosage effect, restricted to smokers, showed the same result with several associations with the high frequencies.

The effect of smoking on hearing loss has given rise to considerable controversy. In a study of CVD, CV risk factors, and hearing loss in the Framingham cohort, Gates et al. (1993) did not find a significant association between smoking (in packyears) and hearing status. In general, they found hearing loss to be strongly associated with CVD events (coronary heart disease, heart attack, stroke, or intermittent claudication), whereas no significant associations with the risk factors for CVD events (such as smoking) were found. They concluded that CVD events themselves, rather than their triggers (e.g., smoking), lead to hearing loss. Hence, they claimed that previously reported associations between smoking and hearing loss were the result of a confounding effect: smokers had on average a higher incidence of CVD events, and these events lead to hearing loss, resulting in a spurious association between smoking and hearing loss.

As a consequence of our recruitment strategyasking for healthy people and choosing a relatively young age range - the prevalence of CVD in the ARHI population is much lower than in the Framingham heart study. On the other hand, our dataset contains a higher number of smokers. Therefore, as our study had included a relatively large number of smoking subjects without CVD events, we have more power to detect effects of smoking alone with less confounding by CVD events. This enabled us to disentangle the effects of smoking and CVD events on hearing.
Accounting for CVD events when testing the association between smoking and $Z$ scores increased, rather than decreased, the significance level. This indicates that the increased occurrence of CVD events in smokers does not explain the association between smoking and hearing loss. This does not necessarily mean that smoking itself is directly responsible for a decrease in hearing ability. We cannot exclude the possibility that the significant association is because of confounding with unknown factors, such as socioeconomic or educational factors, that are associated with both smoking and hearing loss. It is remarkable though, to see a consistent association across so many of the subsamples.

The effect of CVD events seemed to be more pronounced in the low frequencies. In fact, three of the nine subsamples showed a significant association. In the multivariable analysis, including BMI and smoking, the significance levels of CVD events were only slightly decreased compared to the univariable analysis (Supplementary Table 3).

A weak but significant association between smoking and hearing loss in the elderly has been reported previously by Rosenhall et al. (1993) in a cohort of people aged between 70 and 85 years. The effect was only found in males, not in females, suggesting that the effect of smoking was sex-dependent. In our dataset, we have tested for effect modification by gender, but did not observe a significant interaction term in any of the subsamples, despite the fact that our study has higher power. This implies that the effect of smoking was not significantly different between males and females. Although we cannot rule out that the difference in gender effect between the study of Rosenhall et al. and our study can be attributed to the difference in age range between the two studies, reanalysis of our data seems to indicate that it was merely because of the statistical analysis technique used by Rosenhall et al. They performed a split analysis, testing males and females separately for association, whereas in our study, we have performed a single analysis on the entire sample set, including gender as a covariate. It is interesting to note that by performing a split analysis on our data and reanalysing the association between $Z_{\text {high }}$ and packyears in males and females separately, the results were similar to the findings of Rosenhall et al. In three of the subsamples, we found a significant effect in males but not in females. The most likely explanation for the different results between the two statistical analysis techniques is that the nonsignificant results for women in the split analysis were because of a decrease in power. Although all subsamples contained similar numbers of males and females and similar numbers of smokers and nonsmokers, the proportion of smokers is quite different between the sexes: in all subsamples, approximately one third of the females 
ever smoked compared to two thirds in males. Thus, the analysis of women may have lacked power to detect small effects in the separate subsamples. In line with this, the joint analysis with higher sample numbers resulted in a highly significant association in both males and females. Hence, including both males and females into a single analysis, accounting for gender, should be robust to confounding effects of gender and has more power to detect small effects than a split analysis.

Previous reports have shown an association between hearing impairment and chronic alcohol abuse (Rosenhall et al. 1993). On the other hand, a protective effect of moderate alcohol consumption on hearing had been noted before in some, but not all, studies (Brant et al. 1996; Popelka et al. 2000; Itoh et al. 2001; Helzner et al. 2005). Our study could confirm this protective effect. Although our analyses only reached significance in 3/9 subsamples, the direction of the association was consistent across all subsamples, in both the high and the low frequencies. It has been suggested before that the cardioprotective effect of moderate alcohol intake extends to a decreased risk of hearing loss. We also tested whether the effect of alcohol consumption was gender dependent, as effect modification by gender had been noted in previous studies (Helzner et al. 2005). Our joint analysis produced no significant effect modification by gender, and analysis of the separate subsamples did not show a consistent effect modification.

Hearing loss has always been considered an inevitable part of aging, but more and more studies seem to indicate that this is not necessarily true. Apparently, a healthy lifestyle can be beneficial for hearing conservation at higher ages. It is commonly known that a healthy lifestyle protects against CVD. In this study, we have observed a remarkable parallelism between the risk factors for ARHI and those for CVD with similar roles for smoking, high BMI, and regular moderate alcohol consumption.

\section{ACKNOWLEDGEMENTS}

The authors would like to express their sincerest gratitude toward all the volunteers who participated in this study. This work was supported by grants from the European Community (5th Framework project QLRT-2001-00331), the British Royal National Institute for Deaf and Hard of Hearing Individuals (RNID), the Flemish Organization for Scientific Research (FWO-Vlaanderen), and of the University of Antwerp (TOP project).

\section{REFERENCES}

Barrenas ML, Jonsson B, Tuvemo T, Hellstrom PA, Lundgren M. High risk of sensorineural hearing loss in men born small for gestational age with and without obesity or height catch-up growth: A prospective longitudinal register study on birth size in 245,000 Swedish conscripts. J. Clin. Endocrinol. Metab 90:44524456, 2005

Brant LJ, Gordon-Salant S, Pearson JD, Klein Ll, Morrell CH, Metter EJ, Fozard JL. Risk factors related to age-associated hearing loss in the speech frequencies. J. Am. Acad. Audiol 7:152-160, 1996.

Burr H, Lund SP, Sperling BB, Kristensen TS, Poulsen OM. Smoking and height as risk factors for prevalence and 5-year incidence of hearing loss. A questionnaire-based follow-up study of employees in Denmark aged 18-59years exposed and unexposed to noise. Int. J. Audiol 44:531-539, 2005.

Chobanian AV, Bakris GL, Black HR, Cushman WC, Green LA, Izzo JL, Jr, Jones DW, Materson BJ, Oparil S, Wright JT, Jr, Roccella EJ. The Seventh Report of the Joint National Committee on Prevention, Detection, Evaluation, and Treatment of High Blood Pressure: The JNC 7 report. JAMA 289:2560-2572, 2003.

CLARK WW. Noise exposure from leisure activities: A review. J. Acoust. Soc. Am 90:175-181, 1991.

Cruickshanks KJ, Klein R, Klein BE, Wiley TL, Nondahl DM, Tweed TS. Cigarette smoking and hearing loss: The epidemiology of hearing loss study. JAMA 279:1715-1719, 1998.

Davis A. Prevalence of hearing impairment. In: Davis A (ed) Hearing in Adults. London, Whurr, pp. 43-321, 1994.

DeStefano Al, Gates GA, Heard-Costa N, Myers RH, Baldwin CT. Genomewide linkage analysis to presbycusis in the Framingham Heart Study. Arch. Otolaryngol. Head Neck Surg 129:285-289, 2003.

DoвIE RA. Estimating noise-induced permanent threshold shift from audiometric shape: The ISO-1999 model. Ear Hear 26:630-635, 2005.

Engdahl B, Tambs K, Borchgrevink HM, Hoffman HJ. Screened and unscreened hearing threshold levels for the adult population: Results from the Nord-Trondelag Hearing Loss Study. Int. J. Audiol 44:213-230, 2005.

Ferrite S, SANTANa V. Joint effects of smoking, noise exposure and age on hearing loss. Occup. Med. (Lond) 55:48-53, 2005.

Fransen E, Van Laer L, Lemkens N, Caethoven G, Flothmann K, Govaerts P, VAn de Heyning P, VAn Camp G. A novel Z-score-based method to analyze candidate genes for age-related hearing impairment. Ear Hear 25:133-141, 2004.

Fuente A, McPherson B. Central auditory processing effects induced by solvent exposure. Int. J. Occup. Med. Environ. Health 20:271-279, 2007.

Garringer HJ, Pankratz ND, Nichols WC, Reed T. Hearing impairment susceptibility in elderly men and the DFNA18 locus. Arch. Otolaryngol. Head Neck Surg 132:506-510, 2006.

Gates GA, Cobb JL, D'Agostino RB, Wolf PA. The relation of hearing in the elderly to the presence of cardiovascular disease and cardiovascular risk factors. Arch. Otolaryngol. Head Neck Surg 119:156-161, 1993.

Gates GA, Couropmitree NN, Myers RH. Genetic associations in agerelated hearing thresholds. Arch. Otolaryngol. Head Neck Surg 125:654-659, 1999.

Gates GA, Schmid P, Kujawa SG, Nam B, D’Agostino R. Longitudinal threshold changes in older men with audiometric notches. Hear. Res 141:220-228, 2000.

Helzner EP, Cauley Ja, Pratt SR, Wisniewski SR, Zmuda JM, Talbott EO, de Rekeneire N, Harris TB, Rubin SM, Simonsick EM, Tylavski FA, Newman AB. Race and sex differences in age-related hearing loss: The Health, Aging and Body Composition Study. J. Am. Geriatr. Soc 53:2119-2127, 2005.

InTERNATIONAL ORGANISATION OF STANDARDIZATION (1984). International Standard ISO 7029. Acoustics - Threshold of hearing by air conduction as a function of age and sex for otologically normal persons.

Itoh A, Nakashima T, Arao H, Wakat K, Tamakoshi A, Kawamura T, OHNo Y. Smoking and drinking habits as risk factors for hearing 
loss in the elderly: Epidemiological study of subjects undergoing routine health checks in Aichi, Japan. Public Health 115:192196, 2001.

Karlsson KK, Harris JR, Svartengren M. Description and primary results from an audiometric study of male twins. Ear Hear 18:114-120, 1997.

KujaWA SG, Liberman MC. Acceleration of age-related hearing loss by early noise exposure: Evidence of a misspent youth. J. Neurosci 26:2115-2123, 2006.

Kurien M, Thomas K, Bhanu TS. Hearing threshold in patients with diabetes mellitus. J. Laryngol. Otol 103:164-168, 1989.

Lee FS, Matthews LJ, Dubno JR, Mills JH. Longitudinal study of pure-tone thresholds in older persons. Ear Hear 26:1-11, 2005.

Lutman ME, Davis AC. The distribution of hearing threshold levels in the general population aged 18-30years. Audiology 33:327$350,1994$.

Lutman ME, Spencer HS. Occupational noise and demographic factors in hearing. Acta Otolaryngol. Suppl 476:74-84, 1990.

Nomura K, Nakao M, Yano E. Hearing loss associated with smoking and occupational noise exposure in a Japanese metal working company. Int. Arch. Occup. Environ. Health 78:178-184, 2005.

Popelka MM, Cruickshanks KJ, Wiley TL, Tweed TS, Klein BE, Klein R, NONDAHL DM. Moderate alcohol consumption and hearing loss: A protective effect. J. Am. Geriatr. Soc 48:1273-1278, 2000.

Robinson DW. Threshold of hearing as a function of age and sex for the typical unscreened population. Br. J. Audiol 22:5-20, 1988.

Rosenhall U, Sixt E, Sundh V, Svanborg A. Correlations between presbyacusis and extrinsic noxious factors. Audiology 32:234243, 1993.

Sliwinska-Kowalska M, Zamyslowska-Szmytke E, Szymczak W, Kotylo P, Fiszer M, Wesolowski W, Pawlaczyk-Luszczynska M. Ototoxic effects of occupational exposure to styrene and co-exposure to styrene and noise. J. Occup. Environ. Med 45:15-24, 2003.

Sliwinska-Kowalska M, Zamyslowska-Szmytke E, Szymczak W, Kotylo P, Fiszer M, Wesolowski W, Pawlaczyk-Luszczynska M, Bak M, GajdaSzadkowsKa A. Effects of coexposure to noise and mixture of organic solvents on hearing in dockyard workers. J. Occup. Environ. Med 46:30-38, 2004.
Stewart M, Konkle DF, Simpson TH. The effect of recreational gunfire noise on hearing in workers exposed to occupational noise. Ear Nose Throat J 80:32-34, 2001, 36, 38-40.

Torre P, 3rd, Cruickshanks KJ, Klein BE, Klein R, Nondahl DM. The association between cardiovascular disease and cochlear function in older adults. J. Speech Lang. Hear Res 48:473-481, 2005.

Uchida Y, Nakashimat T, Ando F, Nino N, Shimokata H. Is there a relevant effect of noise and smoking on hearing? A populationbased aging study. Int. J. Audiol 44:86-91, 2005.

Ünal M, Tamer L, Dogruer ZN, Yildirim H, Vayisoglu Y, Camdeviren H. N-acetyltransferase 2 gene polymorphism and presbycusis. Laryngoscope 115:2238-2241, 2005.

Van Eyken E, Van Laer L, Fransen E, Topsakal V, Lemkens N, Laureys W, Nelissen N, Vandevelde A, Wienker T, Van De Heyning P, Van CAMP G. KCNQ4: A gene for age-related hearing impairment? Human Mutat 27:1007-1016, 2006.

Van Eyken E, Van Camp G, Fransen E, Topsakal V, Hendrickx JJ, Demeester K, Van de Heyning P, Maki-Torkko E, Hannula S, Sorri M, Jensen M, Parving A, Bille M, Baur M, Pfister M, Bonaconsa A, Mazzoli M, Orzan E, Espeso A, Stephens D, Verbruggen K, Huyghe J, Dhooghe I, Huygen P, Kremer H, Cremers C, Kunst S, Manninen M, Pyykko I, DiazLacava A, Steffens M, Wienker T, Van Laer L. The N-acetyltransferase 2 polymorphism NAT $2 * 6 \mathrm{~A}$ is a causative factor for age related hearing impairment. J. Med. Genet 44:570-578, 2007.

Van Laer L, Van Eyken E, Fransen E, Huyghe JR, Topsakal V, Hendrickx JJ, Hannula S, Maki-Torkko E, Jensen M, Demeester K, Baur M, Bonaconsa A, Mazzoli M, Espeso A, Verbruggen K, Huyghe J, Huygen P, Kunst S, Manninen M, Konings A, DiazLacava AN, Steffens M, Wienker T, Pyykko I, Cremers CW, Kremer H, Dhooge I, Stephens D, Orzan E, Pfister M, Bille M, Parving A, Sorri M, Van de Heyning PH, Van Camp G. The grainyhead like 2 gene (GRHL2), alias TFCP2L3, is associated with age-related hearing impairment. Hum. Mol. Genet 17:159-169, 2008.

Vaughan N, James K, McDermott D, Griest S, Fausti S. A 5-year prospective study of diabetes and hearing loss in a veteran population. Otol. Neurotol 27:37-43, 2006.

Wild DC, Brewster MJ, Banerjee AR. Noise-induced hearing loss is exacerbated by long-term smoking. Clin. Otolaryngol 30:517-520, 2005. 